\title{
A remark on the set of arbitrage-free prices in a multi-period model
}

\section{Ranjan, Abhishek}

Published in:

International Journal of Economic Theory

Publication date:

2013

Link back to DTU Orbit

Citation (APA):

Ranjan, A. (2013). A remark on the set of arbitrage-free prices in a multi-period model. International Journal of Economic Theory, 9, 35-43.

\section{General rights}

Copyright and moral rights for the publications made accessible in the public portal are retained by the authors and/or other copyright owners and it is a condition of accessing publications that users recognise and abide by the legal requirements associated with these rights.

- Users may download and print one copy of any publication from the public portal for the purpose of private study or research.

- You may not further distribute the material or use it for any profit-making activity or commercial gain

- You may freely distribute the URL identifying the publication in the public portal

If you believe that this document breaches copyright please contact us providing details, and we will remove access to the work immediately and investigate your claim. 
doi: 10.1111/j.1742-7363.2012.12004.x

\title{
A remark on the set of arbitrage-free prices in a multi-period model
}

\author{
Bernard Cornet* and Abhishek Ranjan ${ }^{\dagger}$
}

\begin{abstract}
We study the convexity property of the set $Q_{\mathcal{F}}$ of arbitrage-free prices of a multi-period financial structure $\mathcal{F}$. The set of arbitrage-free prices is shown to be a convex cone under conditions on the financial structure $\mathcal{F}$ that hold in particular for short-lived assets. Furthermore, we provide examples of equivalent financial structures $\mathcal{F}$ and $\mathcal{F}^{\prime}$ such that $Q_{\mathcal{F}}$ is a convex cone, but $Q_{\mathcal{F}^{\prime}}$ is neither convex nor a cone.
\end{abstract}

Key words arbitrage-free prices, convexity, equivalent financial structure, multi-period model

JEL classification C02, D53

Accepted 11 September 2012

\section{Introduction}

The two-date model is not sufficient to capture the time evolution of realistic models, as there are many properties which hold in a two-date model but not in a multi-period model with long-lived assets. Similarly the multi-period financial model with short-lived assets is very close to the two-date model and does not capture the full realism of the multi-period model with long-lived assets.

In this paper, we study the convexity property of the set $Q_{\mathcal{F}}$ of arbitrage-free prices of a multiperiod financial structure $\mathcal{F}$, which always hold in a multi-period financial model with short-lived assets but may not hold with long-lived assets. Our first result shows that the set $Q_{\mathcal{F}}$ of arbitrage-free prices is a convex cone under a sufficient condition that holds in particular for short-lived assets (hence also in the two-date model). Furthermore, we provide examples of "equivalent" financial structures $\mathcal{F}$ and $\mathcal{F}^{\prime}$ such that $Q_{\mathcal{F}}$ is a convex cone, but $Q_{\mathcal{F}^{\prime}}$ is neither convex nor a cone.

The property that the set $Q_{\mathcal{F}}$ of arbitrage-free prices is a convex cone is a key property for the existence of financial equilibria, as shown in Cornet and Ranjan (2012a, 2012b) and in Aouani and Cornet (2009). Moreover, if the financial exchange economy $(\mathcal{E}, \mathcal{F})$ does not satisfy the property that the set $Q_{\mathcal{F}}$ of arbitrage-free prices is a convex cone, we can aim to find an equivalent financial structure $\mathcal{F}^{\prime}$ such that $Q_{\mathcal{F}^{\prime}}$ is a convex cone. Since, $(\mathcal{E}, \mathcal{F})$ and $\left(\mathcal{E}, \mathcal{F}^{\prime}\right)$ have the same consumption equilibria when $\mathcal{F}$ and $\mathcal{F}^{\prime}$ are equivalent, we can thus deduce the existence of financial equilibria of $(\mathcal{E}, \mathcal{F})$ from the existence of equilibria of $\left(\mathcal{E}, \mathcal{F}^{\prime}\right)$.

The paper is organized as follows. In Section 1, we present the multi-period model of time and uncertainty, together with the financial structure with long-lived assets, and we recall the version of the fundamental theorem of asset pricing that allows us to characterize arbitrage-free prices by the

\footnotetext{
Paris School of Economics, Université Paris 1, France and University of Kansas, USA. Email: cornet@univ-paris1.fr, cornet@ku.edu

$\dagger \quad$ Paris School of Economics, Université Paris 1, France.
} 
existence of positive prices at every node. In Section 2, we provide sufficient conditions for $Q_{\mathcal{F}}$ to be a convex cone and we show that these conditions hold in particular for short-lived assets (hence also in the two-date model). Then we exhibit examples of financial structures $\mathcal{F}$ for for which $Q_{\mathcal{F}}$ is neither convex nor a cone. Finally, we show that the property of $Q_{\mathcal{F}}$ being a convex cone is not preserved when $\mathcal{F}$ is replaced by an equivalent financial structure $\mathcal{F}^{\prime}$ and the formal definition of equivalence is provided. The proofs of some results are postponed to the Appendix.

\section{The model}

\subsection{Time and uncertainty in a multi-period model}

We consider a multi-period financial exchange economy with $T+1$ dates, $t \in \mathcal{T}:=\{0, \ldots, T\}$. The stochastic structure of the model is described by a finite-tree $\mathcal{D}$ of length $T$, and we shall essentially use the same notation as in Debreu (1959) and Magill and Quinzii (1996) to whom we refer for an equivalent presentation with information partitions. The set $\mathcal{D}_{t}$ denotes the set of nodes (also called date events) that may occur at date $t$ and the family $\left(\mathcal{D}_{t}\right)_{t \in \mathcal{T}}$ defines a partition of the set $\mathcal{D}$.

At each date $t \neq T$, there is a priori uncertainty about which node will prevail at the next date. There is a unique non-stochastic event occurring at date $t=0$, which is simply denoted 0 , so $\mathcal{D}_{0}=\{0\}$. Every node $\xi \neq 0$ has a unique immediate predecessor, denoted $\xi^{-}$. For each $\xi \in \mathcal{D}$, we define $\xi^{+}=\left\{\bar{\xi} \in \mathcal{D}: \xi=\bar{\xi}^{-}\right\}$as the set of immediate successors of $\xi$, and by the definition of the date-event tree, the set $\xi^{+}$is non-empty if and only if $\xi \in \mathcal{D} \backslash \mathcal{D}_{T}$. Moreover, we define the set of successors (not necessarily immediate) of $\xi$ as

$$
\mathcal{D}^{+}(\xi)=\left\{\xi^{\prime} \in \mathcal{D}: \exists\left(\xi_{1}, \xi_{2}, \ldots, \xi_{k}\right), \xi_{k}=\xi^{\prime-}, \xi_{k-1}=\xi_{k}^{-}, \ldots, \xi_{1}=\xi_{2}^{-}, \xi=\xi_{1}^{-}\right\}
$$

We also use the notation $\xi^{\prime}>\xi\left(\xi^{\prime} \geq \xi\right)$ if $\xi^{\prime} \in \mathcal{D}^{+}(\xi)\left(\xi^{\prime} \in \mathcal{D}^{+}(\xi) \cup\{\xi\}\right)$.

\subsection{The financial structure}

We consider a financial structure $\mathcal{F}$ with a set $J=\{1, \ldots, J\}$ of assets; every asset $j \in J$ is characterized by the pair $\left(\xi^{j}, V^{j}\right) \in \mathcal{D} \times \mathbb{R}^{\mathcal{D}}$, where $\xi^{j}$ is the emission node of asset $j$ and $V^{j} \in \mathbb{R}^{\mathcal{D}}$ is its payoff. We will adopt the convention that $V_{\xi}^{j}$, the payoff of asset $j$ at node $\xi$, is defined for every $\xi$ and we will thus assume that the payoff is zero at every node which does not succeed $\xi^{j}$, that is, $V_{\xi}^{j}=0$ if $\xi \notin \mathcal{D}^{+}\left(\xi^{j}\right)$. The financial structure $\mathcal{F}$ can be summarized as $\mathcal{F}=\left(J,\left(\xi^{j}, V^{j}\right)_{j \in J}\right)$.

We denote by $q=\left(q^{1}, q^{2}, \ldots, q^{J}\right)$ the prices of the assets, by $W^{j}(\cdot): \mathbb{R} \rightarrow \mathbb{R}^{\mathcal{D}}$ the total payoff matrix of the asset $j$ defined as follows $W^{j}\left(q^{j}\right)=V^{j}-q^{j} \mathbb{I}_{\xi j}$, and by $W_{\mathcal{F}}(\cdot)=\left[W^{1}(\cdot), \ldots, W^{J}(\cdot)\right]$ the total payoff mapping of $\mathcal{F}$, thus a mapping from $\mathbb{R}^{J}$ to $\mathbb{R}^{\mathcal{D} \times J}$. We note that there is one-to-one correspondence between $\mathcal{F}$ and $W_{\mathcal{F}}(\cdot)$; indeed $W_{\mathcal{F}}(\cdot)$ is well defined from $\mathcal{F}$, and conversely from $W_{\mathcal{F}}(\cdot)$ the formulas $\left(V^{1}, \ldots, V^{J}\right)=W_{\mathcal{F}}(0)$ and $\mathbb{I}_{\xi^{j}}=W^{j}(0)-W^{j}(1)$ uniquely define the payoffs of the assets and its emission dates. In the following, we indifferently summarize the financial structure by

$$
\mathcal{F}=\left(J,\left(\xi^{j}, V^{j}\right)_{j \in J}\right) \text { or } W_{\mathcal{F}}(\cdot)
$$




\subsection{Arbitrage-free prices}

Let $q \in \mathbb{R}^{I}$; we say that $(\mathcal{F}, q)$ is arbitrage-free if there exists no portfolio $z \in \mathbb{R}^{I}$ such that $W_{\mathcal{F}}(q) z>0$, or equivalently $\left[W_{\mathcal{F}}(q)\left(\mathbb{R}^{J}\right)\right] \cap \mathbb{R}_{+}^{\mathcal{D}}=\{0\}$. The set of arbitrage-free prices is denoted by $Q_{\mathcal{F}}$.

In this framework, the fundamental theorem of asset pricing (see Angeloni and Cornet 2006) stating that $(\mathcal{F}, q)$ is arbitrage-free for the financial structure $\mathcal{F}$ if and only if there exists $\lambda \in \mathbb{R}_{++}^{D}$ satisfying $W_{\mathcal{F}}^{T}(q) \lambda=0$, that is, for all $j \in J$,

$$
\lambda W^{j}(q)=\lambda\left[V^{j}-q^{j} 1_{\xi^{j}}\right]=0 \text { if and only if } \lambda\left(\xi^{j}\right) q^{j}=\lambda V^{j} .
$$

It is important to notice that the above equations uniquely define $q^{j}$, for given $\lambda \in \mathbb{R}_{++}^{D}$. So, for every $j$ we define

$$
q_{\mathcal{F}}^{j}(\lambda):=\left(1 /\left(\lambda\left(\xi^{j}\right)\right) V^{j} \lambda \text { and } q_{\mathcal{F}}(\lambda)=\left(q_{\mathcal{F}}^{1}(\lambda), \ldots, q_{\mathcal{F}}^{J}(\lambda)\right),\right.
$$

and we notice that for every $\lambda \in \mathbb{R}_{++}^{D}$,

$$
W_{\mathcal{F}}\left(q_{\mathcal{F}}(\lambda)\right)^{T} \lambda=0 \text {, that is, } \lambda W^{j}\left(q_{\mathcal{F}}(\lambda)\right)=0 \text { for all } j .
$$

\section{The main results}

\subsection{Convexity results}

Our first result provides a condition under which the set of arbitrage-free prices $Q_{\mathcal{F}}$ is a convex cone.

Proposition 1 The set $Q_{\mathcal{F}}$ is a convex cone if the financial structure $\mathcal{F}$ satisfies:

$$
V_{\xi}^{j} V_{\xi}^{k}=0 \text { for all } \xi \text {, whenever } \xi^{j} \neq \xi^{k} .
$$

We recall that an asset $j$ is said to be short-lived if it has non-zero payoffs only at the immediate successors of the node at which it is issued, that is, $V_{\xi}^{j}=0$ for all $\xi \notin\left(\xi^{j}\right)^{+}$. We also recall that an Arrow security is an asset with a payoff of 1 at a unique node in the future $(t \neq 0)$, and a zero payoff at all other nodes.

Corollary The set $Q_{\mathcal{F}}$ is a convex cone under each of the following conditions:

(i) $\mathcal{F}$ has only short-lived assets;

(ii) $\mathcal{F}$ is a two-date financial structure, that is, $\mathcal{T}=\{0,1\}$;

(iii) $\mathcal{F}$ consists only of Arrow securities with payoffs at different nodes.

The proof of the corollary is immediate and we now give the proof of Proposition 1.

Proof: $Q_{\mathcal{F}}$ is a cone. $Q_{\mathcal{F}}$ is cone, that is, for all $q \in Q_{\mathcal{F}}$ and $\alpha \in(0, \infty), \alpha q \in Q_{\mathcal{F}}$. Since $q \in Q_{\mathcal{F}}$, from the characterization of arbitrage-free prices, there exists $\lambda \in \mathbb{R}_{++}^{\mathcal{D}}$ satisfying

$$
\lambda\left(\xi^{j}\right) q^{j}=\sum_{\xi>\xi^{j}} \lambda(\xi) V_{\xi}^{j}, \quad \forall j \in J
$$


Now to prove that $\alpha q \in Q_{\mathcal{F}}$, we need to construct $\lambda^{\prime} \in \mathbb{R}_{++}^{\mathcal{D}}$ such that:

$$
\lambda^{\prime}\left(\xi^{j}\right) \alpha q^{j}=\sum_{\xi>\xi^{j}} \lambda^{\prime}(\xi) V_{\xi}^{j}, \quad \forall j \in J
$$

We proceed by induction on the set $t \in\{0,1,2, \ldots, T\}$ and we define $\lambda^{\prime}(\xi)$ on $\mathcal{D}=\cup_{t \in \mathcal{T}} \mathcal{D}_{t}$ as follows. We take $\lambda^{\prime}(0)=1$, so $\lambda^{\prime}$ is defined on the set $\mathcal{D}_{0}$. Assume that $\lambda^{\prime}(\xi)$ is defined on $\cup_{\tau \leq t} \mathcal{D}_{\tau}$ for $t \leq T-1$, then we define $\lambda^{\prime}(\xi)$ for $\xi \in \mathcal{D}_{t+1}$ as follows. Either, for all $k \in J, V_{\xi}^{k}=0$, then we define $\lambda^{\prime}(\xi)=1$. Or there exist at least one $k \in J$, such that $V_{\xi}^{k} \neq 0$; then from assumption (1), we know that $\xi^{k}=\xi^{k^{\prime}}$ for all asset $k^{\prime} \in J$ such that $V_{\xi}^{k^{\prime}} \neq 0$. So we can define $\lambda^{\prime}(\xi)=\frac{\lambda(\xi)}{\lambda\left(\xi^{k}\right)} \lambda^{\prime}\left(\xi^{k}\right)$ since $\xi^{k} \in D^{-}(\xi) \subset \cup_{\tau \leq t} \mathcal{D}_{\tau}$.

To summarize, $\lambda^{\prime}$ is defined as:

$$
\lambda^{\prime}(\xi)= \begin{cases}\alpha \frac{\lambda(\xi)}{\lambda\left(\xi^{k}\right)} \lambda^{\prime}\left(\xi^{k}\right) & \text { if there exist } k \text { satisfying } V_{\xi}^{k} \neq 0, \\ 1 & \text { otherwise. }\end{cases}
$$

Clearly, whenever $V_{\xi}^{j} \neq 0$, we have $\frac{\lambda^{\prime}(\xi)}{\lambda^{\prime}\left(\xi^{j}\right)}=\alpha \frac{\lambda(\xi)}{\lambda\left(\xi^{j}\right)}$. Therefore,

$$
\lambda^{\prime}\left(\xi^{j}\right) \alpha q^{j}=\sum_{\xi>\xi^{j}} \lambda^{\prime}(\xi) V_{\xi}^{j}, \quad \forall j \in J
$$

Consequently, $\alpha q \in Q_{\mathcal{F}}$, which ends the proof that $Q_{\mathcal{F}}$ is a cone.

$Q_{\mathcal{F}}$ is convex. Since $Q_{\mathcal{F}}$ is a cone, it suffices to prove that $q+r \in Q_{\mathcal{F}}$, whenever $q, r$ are in $Q_{\mathcal{F}}$. Indeed, let $q \in Q_{\mathcal{F}}, r \in Q_{\mathcal{F}}$; then there exist $\mu \in \mathbb{R}_{++}^{\mathcal{D}}, v \in \mathbb{R}_{++}^{\mathcal{D}}$ such that

$$
q^{j}=\sum_{\xi>\xi^{j}} \frac{\mu(\xi)}{\mu\left(\xi^{j}\right)} V_{\xi}^{j} \text { and } r^{j}=\sum_{\xi>\xi^{j}} \frac{v(\xi)}{v\left(\xi^{j}\right)} V_{\xi}^{j}, \quad \forall j \in J
$$

Therefore, $q^{j}+r^{j}=\sum_{\xi>\xi^{j}}\left(\frac{\mu(\xi)}{\mu\left(\xi^{j}\right)}+\frac{v(\xi)}{v\left(\xi^{j}\right)}\right) V_{\xi^{j}}^{j}$

Using the same argument as in the case of a cone, we can construct $\lambda \in \mathbb{R}_{++}^{\mathcal{D}}$ by induction so that

$$
\lambda(\xi)= \begin{cases}\left(\frac{\mu(\xi)}{\mu\left(\xi^{k}\right)}+\frac{v(\xi)}{v\left(\xi^{k}\right)}\right) \lambda\left(\xi^{j}\right) & \text { if there exist } k \text { satisfying } V_{\xi}^{k} \neq 0 \\ 1 & \text { otherwise. }\end{cases}
$$

If $V_{\xi}^{j} \neq 0$, then $\frac{\lambda(\xi)}{\lambda\left(\xi^{j}\right)}=\left(\frac{\mu(\xi)}{\mu\left(\xi^{j}\right)}+\frac{v(\xi)}{v\left(\xi^{j}\right)}\right)$. Therefore, $\lambda\left(\xi^{j}\right)\left(q^{j}+r^{j}\right)=\sum_{\xi>\xi^{j}} \lambda(\xi) V_{\xi}^{j}$. Consequently, $q+r \in Q_{\mathcal{F}}$ which implies $Q_{\mathcal{F}}$ is convex.

We now give a second convexity result. Consider a financial structure $\mathcal{F}$ with one asset issued at time $t=0$, with payoff $V^{0}$ and price $q^{0}$, and other assets issued at future times. We denote the financial structure "starting" tomorrow by $\mathcal{F}^{\prime}$; then the total payoff matrix for $\mathcal{F}$ is given by

$$
W_{\mathcal{F}}\left(q^{0}, q^{\prime}\right):=\left[\begin{array}{cc}
-q^{0} & 0 \\
V^{0} & W_{\mathcal{F}^{\prime}}\left(q^{\prime}\right)
\end{array}\right] .
$$


Proposition 2 If the set $Q_{\mathcal{F}^{\prime}}$ is a convex cone, and either $V^{0} \geq 0$ or $V^{0} \leq 0$, then the set $Q_{\mathcal{F}}$ is also a convex cone. Proof: Step 1: $V^{0}=0$. Clearly, $Q_{\mathcal{F}}=\{0\} \times Q_{\mathcal{F}^{\prime}}$, which is a convex cone.

Step 2: $V^{0} \geq 0$ and $V^{0} \neq 0$. Now we claim that $Q_{\mathcal{F}}=\mathbb{R}_{++} \times Q_{\mathcal{F}^{\prime}}$, which clearly implies that $Q_{\mathcal{F}}$ is a convex cone.

We first prove that $Q_{\mathcal{F}} \subset \mathbb{R}_{++} \times Q_{\mathcal{F}^{\prime}}$. From the characterization theorem of arbitrage-free prices, for every $q=\left(q^{0}, q^{\prime}\right) \in Q_{\mathcal{F}}$, there exist $\lambda=\left(\lambda_{0}, \lambda^{\prime}\right) \gg 0$, such that

$$
W_{\mathcal{F}}\left(q_{0}, q^{\prime}\right)^{T} \lambda:=\left[\begin{array}{c}
-q^{0} \lambda_{0}+V^{0} \lambda^{\prime} \\
W_{\mathcal{F}^{\prime}}\left(q^{\prime}\right)^{T} \lambda^{\prime}
\end{array}\right]=\left[\begin{array}{l}
0 \\
0
\end{array}\right] .
$$

Therefore, $q^{0}=V^{0} \cdot \lambda^{\prime} / \lambda_{0}>0$, and $W_{\mathcal{F}^{\prime}}\left(q^{\prime}\right)^{T} \lambda^{\prime}=0$ implies $q^{\prime} \in Q_{\mathcal{F}^{\prime}}$ (from the characterization theorem of arbitrage-free prices). Therefore $q \in \mathbb{R}_{++} \times Q_{\mathcal{F}^{\prime}}$.

We now prove that $\mathbb{R}_{++} \times Q_{\mathcal{F}^{\prime}} \subset Q_{\mathcal{F}}$. Let $q=\left(q^{0}, q^{\prime}\right) \in \mathbb{R}_{++} \times Q_{\mathcal{F}^{\prime}}$. From the characterization theorem of arbitrage-free prices, since $q^{\prime} \in Q_{\mathcal{F}^{\prime}}$, there exist $\lambda^{\prime} \gg 0$ such that $W_{\mathcal{F}^{\prime}}\left(q^{\prime}\right)^{T} \lambda^{\prime}=0$. Now we choose $\lambda_{0}=V^{0} \lambda^{\prime} / q^{0}>0$, and we notice that $\lambda=\left(\lambda_{0}, \lambda^{\prime}\right) \gg 0$ satisfies $W_{\mathcal{F}}(q)^{T} \lambda=0$. Therefore, $q \in Q_{\mathcal{F}}$. Hence, $\mathbb{R}_{++} \times Q_{\mathcal{F}^{\prime}}=Q_{\mathcal{F}}$.

Step 3: $V^{0} \leq 0$ and $V^{0} \neq 0$. The proof is similar to that of Step 2 .

\subsection{Examples of non-convexity}

The following proposition provides examples for which $Q_{\mathcal{F}}$ is neither convex nor a cone.

Proposition 3 Consider the financial structure $\mathcal{F}$ with three dates and two assets, the first one issued at $t=0$ and the second one at $t=1$, with total payoff matrix

$$
W_{\mathcal{F}}(q):=\left[\begin{array}{cc}
-q^{1} & 0 \\
a & -q^{2} \\
b & 1
\end{array}\right] .
$$

The set $Q_{\mathcal{F}}$ is a convex cone if and only if $a b \geq 0$.

The proof is a direct consequence of the following claim. ${ }^{1}$

Claim 1 We have

$$
Q_{\mathcal{F}}(a, b)= \begin{cases}\{0\} \times \mathbb{R}_{++} & \text {if }(a, b)=(0,0), \\ \mathbb{R}_{--} \times \mathbb{R}_{++} & \text {if } a \leq 0, b \leq 0 \text { and }(a, b) \neq(0,0), \\ {\left[\mathbb{R}_{--} \times\left(0,-\frac{a}{b}\right)\right] \cup\left[\mathbb{R}_{++} \times\left(-\frac{a}{b}, \infty\right)\right] \cup\left\{\left(0,-\frac{a}{b}\right)\right\}} & \text { if } a<0 \text { and } b>0, \\ {\left[\mathbb{R}_{--} \times\left(-\frac{a}{b}, \infty\right)\right] \cup\left[\mathbb{R}_{++} \times\left(0,-\frac{a}{b}\right)\right] \cup\left\{0,-\frac{a}{b}\right\}} & \text { if } a>0 \text { and } b<0, \\ \mathbb{R}_{++} \times \mathbb{R}_{++} & \text {if } a \geq 0, b \geq 0 \text { and }(a, b) \neq(0,0) .\end{cases}
$$

1 Note also that if $a b \geq 0$, then the set $Q_{\mathcal{F}}$ is a convex cone from Proposition 2. 
The proof of the claim is given in the Appendix. The set $Q_{\mathcal{F}}(a, b)$ is represented in the following figure when it is not convex.

We now give a second example for which $Q_{\mathcal{F}}$ is neither convex nor a cone.

Proposition 4 Consider the financial structure $\mathcal{F}$ with 3 dates, 3 assets, first and second assets issued at $t=0$ and third one at $t=1$ with total payoff matrix

$$
W_{\mathcal{F}}(q):=\left[\begin{array}{ccc}
-q^{1} & -q^{2} & 0 \\
a & c & -q^{3} \\
b & d & 1
\end{array}\right] \text { with } a d-b c \neq 0 .
$$

Then the set $Q_{\mathcal{F}}$ is neither convex nor a cone.

Proof: $Q_{\mathcal{F}}$ is not a cone. From the characterization theorem of arbitrage-free prices, $q \in Q_{\mathcal{F}}$ if and only if there exists $\lambda=\left(1, \lambda_{1}, \lambda_{2}\right) \gg 0$ such that

$$
q^{1}=a \lambda_{1}+b \lambda_{2}, q^{2}=c \lambda_{1}+d \lambda_{2}, \text { and } \lambda_{1} q^{3}=\lambda_{2}
$$

From the above equations (using $a d-b c \neq 0$ ), we get

$$
\begin{aligned}
Q=\{ & \left(q^{1}, q^{2}, q^{3}\right) \mid q^{3}\left(d q^{1}-b q^{2}\right)=a q^{2}-c q^{1} \\
& \left.\left(d q^{1}-b q^{2}\right)(a d-b c)>0, \text { and }\left(a q^{2}-c q^{1}\right)(a d-b c)>0\right\} .
\end{aligned}
$$

Suppose that $Q_{\mathcal{F}}$ is a cone and $q=\left(q^{1}, q^{2}, q^{3}\right) \in Q_{\mathcal{F}}$, then $q^{3}\left(d q^{1}-b q^{2}\right)=a q^{2}-c q^{1}$. Since $Q_{\mathcal{F}}$ is a cone, $\alpha q=\left(\alpha q^{1}, \alpha q^{2}, \alpha q^{3}\right) \in Q_{\mathcal{F}}$ for all $\alpha>0$. Taking $\alpha=1 / 2$, we get $1 / 2=1$, a contradiction. Hence, $Q_{\mathcal{F}}$ is not a cone.

$Q_{\mathcal{F}}$ is not convex. Take $q=\left(q^{1}, q^{2}, q^{3}\right) \in Q_{\mathcal{F}}$ and by simple calculations, we get

$$
q^{1}\left(d q^{3}+c\right)=q^{2}\left(b q^{3}+a\right)
$$

Since $a d-b c \neq 0$, implies either $b \neq 0$ or $d \neq 0$, and without loss of generality we can assume $b \neq 0$. We let $q^{1}=1, q^{\prime 2}=q^{2}-\frac{d}{b}$ and $q^{\prime 3}=b q^{3}+a$ and we notice that the set $\left\{\left(q^{\prime 2}, q^{\prime 3}\right) \mid q^{\prime 2} q^{\prime 3}=\frac{b c-a d}{b}\right\}$ is not convex (recalling that $b \neq 0$, and $b c-a d \neq 0$ ). Hence, $Q_{\mathcal{F}}$ is not convex.
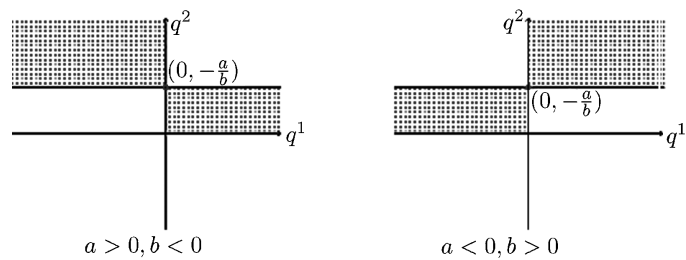

Figure 1 Non-convex set of arbitrage-free prices 


\subsection{Equivalence of financial structures may not preserve convexity}

The main result of this section shows that the property that $Q_{\mathcal{F}}$ is a convex cone may not be preserved when $\mathcal{F}$ is replaced by an equivalent financial structure $\mathcal{F}^{\prime}$. The following proposition recalls the definition of equivalent financial structures:

Proposition 5 Two financial structures $\mathcal{F}$ and $\mathcal{F}^{\prime}$ are equivalent if one of the following two equivalent conditions holds:

(i) $\forall q \in Q_{\mathcal{F}}, \exists q^{\prime}$ such that $\operatorname{Im} W_{\mathcal{F}}(q)=\operatorname{Im} W_{\mathcal{F}^{\prime}}\left(q^{\prime}\right)$, and $\forall q^{\prime} \in Q_{\mathcal{F}^{\prime}}, \exists q$ such that $\operatorname{Im} W_{\mathcal{F}^{\prime}}\left(q^{\prime}\right)=$ $\operatorname{Im} W_{\mathcal{F}}(q)$.

(ii) $\forall \lambda \in \mathbb{R}_{++}^{S}, \operatorname{Im} W_{\mathcal{F}}\left(q_{\mathcal{F}}(\lambda)\right)=\operatorname{Im} W_{\mathcal{F}^{\prime}}\left(q_{\mathcal{F}^{\prime}}(\lambda)\right)$.

The intuition behind this definition is the following. Financial structures allow agents to transfer wealth across nodes of the date-event tree and thereby give them the possibility to enlarge their budget set. The well-known consequence of this definition is that, regardless of the standard exchange economy $\mathcal{E}$, consumption equilibria are the same when agents carry out their financial activities through two different structures $\mathcal{F}$ and $\mathcal{F}^{\prime}$.

Define the financial structures $\mathcal{F}$ and $\mathcal{F}^{\prime}$ by

$$
W_{\mathcal{F}}(q):=\left[\begin{array}{ccc}
-q^{1} & -q^{2} & 0 \\
a & c & -q^{3} \\
b & d & 1
\end{array}\right], W_{\mathcal{F}^{\prime}}\left(q^{\prime}\right):=\left[\begin{array}{cc}
-q^{\prime 1} & 0 \\
1 & -q^{\prime 2} \\
0 & 1
\end{array}\right] .
$$

Proposition 6 If $a d-b c \neq 0$, then the financial structures $\mathcal{F}$ and $\mathcal{F}^{\prime}$ defined by (2) are equivalent, $\mathcal{F}^{\prime}$ has only short-lived assets and hence $Q_{\mathcal{F}^{\prime}}$ is a convex cone, but $Q_{\mathcal{F}}$ is neither convex nor a cone.

The proof of this proposition goes as follows. The set $Q_{\mathcal{F}}$ is neither convex nor a cone by Proposition 4 and $Q_{\mathcal{F}^{\prime}}$ is a convex cone since $\mathcal{F}^{\prime}$ has only short-lived assets by the corollary to Proposition 1.

The proof of the equivalence is given in the Appendix.

\section{Conclusions}

Proposition 6 is interesting in several ways. First, the financial structure $\mathcal{F}$ with long-lived assets has been "simplified" by showing it is equivalent to $\mathcal{F}^{\prime}$, which (i) has less assets; and (ii) all of which are short-lived. Note, however, that this assertion does not hold true in general, as shown by the following example.

Example 1 Consider $\mathcal{F}$ with three dates (one node at each date) and one asset which is issued at time $t=0$, and has payoff 0 and 1 at $t=1$ and $t=2$, respectively.

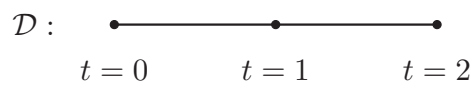

Clearly, $\mathcal{F}$ is not equivalent to any financial structure with short-lived assets. 
The property that $Q_{\mathcal{F}}$ is a convex cone (weaker than $\mathcal{F}$ having only short-lived assets) is satisfied in Example 1, and has some important consequences for the existence problem of equilibria as discussed in the companion paper (Cornet and Ranjan 2012b). Indeed, it is proved that the financial exchange economy $(\mathcal{E}, \mathcal{F})$ has equilibria under standard assumptions together with the assumption that $Q_{\mathcal{F}}$ is a convex cone or that $\mathcal{F}$ is equivalent to some other financial structure $\mathcal{F}^{\prime}$ such that $Q_{\mathcal{F}^{\prime}}$ is a convex cone.

\section{Appendix}

\section{Proof of Claim 1}

We need to show that

$$
Q_{\mathcal{F}}(a, b)= \begin{cases}\{0\} \times \mathbb{R}_{++} & \text {if }(a, b)=(0,0), \\ \mathbb{R}_{--} \times \mathbb{R}_{++} & \text {if } a \leq 0, b \leq 0 \text { and }(a, b) \neq(0,0), \\ {\left[\mathbb{R}_{--} \times\left(0,-\frac{a}{b}\right)\right] \cup\left[\mathbb{R}_{++} \times\left(-\frac{a}{b}, \infty\right)\right] \cup\left\{\left(0,-\frac{a}{b}\right)\right\}} & \text { if } a<0 \text { and } b>0, \\ {\left[\mathbb{R}_{--} \times\left(-\frac{a}{b}, \infty\right)\right] \cup\left[\mathbb{R}_{++} \times\left(0,-\frac{a}{b}\right)\right] \cup\left\{0,-\frac{a}{b}\right\}} & \text { if } a>0 \text { and } b<0, \\ \mathbb{R}_{++} \times \mathbb{R}_{++} & \text {if } a \geq 0, b \geq 0 \text { and }(a, b) \neq(0,0) .\end{cases}
$$

Step 1: $b \neq 0$. From the characterization theorem of arbitrage-free prices, $q \in Q_{\mathcal{F}}(a, b)$ if and only if there exists $\lambda=\left(1, \lambda_{2}, \lambda_{3}\right) \in \mathbb{R}_{++}^{3}$ such that

$$
q^{1}=a \lambda_{2}+b \lambda_{3} \text { and } q^{2} \lambda_{2}=\lambda_{3}
$$

Hence

$$
\begin{aligned}
& q^{1}=a \lambda_{2}+\lambda_{2} q^{2} b=\lambda_{2}\left(a+q^{2} b\right) \text { and } q^{2}=\frac{\lambda_{3}}{\lambda_{2}}>0 . \\
& q^{1}= \begin{cases}<0 & \Leftrightarrow \text { either }\left(q^{2}<\frac{-a}{b} \text { and } b>0\right) \text { or }\left(q^{2}>\frac{-a}{b} \text { and } b<0\right) \text { or }(a<0 \text { and } b=0), \\
=0 & \Leftrightarrow q^{2}=\frac{-a}{b} \text { or }(a=0 \text { and } b=0), \\
>0 & \Leftrightarrow \text { either }\left(q^{2}>\frac{-a}{b} \text { and } b>0\right) \text { or }\left(q^{2}<\frac{-a}{b} \text { and } b<0\right) \text { or }(a>0 \text { and } b=0) .\end{cases}
\end{aligned}
$$

Now we consider the following cases.

(i) $a \leq 0$ and $b<0$. We have $q^{2}>0$ and we want to show that $q_{1}<0$. Assume $q^{1} \geq 0$; then the above equation implies $q^{2} \leq-\frac{a}{b} \leq 0$, a contradiction.

(ii) $a \leq 0$ and $b>0$. From the above equations, under the conditions $b>0$ and $-\frac{a}{b} \geq 0, q^{1}<0$ implies $0<q^{2}<-\frac{a}{b}, q^{1}=0$ implies $q^{2}=-\frac{a}{b}$, and $q^{1}>0$ implies $q^{2}>-\frac{a}{b}$.

(iii) $a \geq 0$ and $b<0$. From the above equations, under the conditions $b<0$ and $-\frac{a}{b} \geq 0, q^{1}<0$ implies $q^{2}>-\frac{a}{b}, q^{1}=0$ implies $q^{2}=-\frac{a}{b}$, and $q^{1}>0$ implies $0<q^{2}<-\frac{a}{b}$.

(iv) $a \geq 0$ and $b>0$. We have $q^{2}>0$ and we want to show that $q_{1}>0$. Assume $q^{1} \leq 0$, then the above equation implies $q^{2} \leq-\frac{a}{b} \leq 0$, a contradiction. 
Step 2: $b=0$. Now we consider the following cases:

(i) $a=0$. Clearly, $Q_{\mathcal{F}}=\{0\} \times \mathbb{R}_{++}$.

(ii) $a<0$. Clearly, $Q_{\mathcal{F}}=\mathbb{R}_{--} \times \mathbb{R}_{++}$.

(iii) $a>0$. Clearly, $Q_{\mathcal{F}}=\mathbb{R}_{++} \times \mathbb{R}_{++}$.

\section{Proof of Proposition 6}

We need to show that $\mathcal{F}$ and $\mathcal{F}^{\prime}$ are equivalent. Let $\lambda=\left(1, \lambda_{1}, \lambda_{2}\right) \gg 0$. Then

$$
W_{\mathcal{F}}(q(\lambda)):=\left[\begin{array}{ccc}
-\left(a \lambda_{1}+b \lambda_{2}\right) & -\left(c \lambda_{1}+d \lambda_{2}\right) & 0 \\
a & c & -\frac{\lambda_{2}}{\lambda_{1}} \\
b & d & 1
\end{array}\right], \quad W_{\mathcal{F}^{\prime}}\left(q^{\prime}(\lambda)\right):=\left[\begin{array}{cc}
-\lambda_{1} & 0 \\
1 & -\frac{\lambda_{2}}{\lambda_{1}} \\
0 & 1
\end{array}\right] .
$$

Now, we claim that $\operatorname{rank} W_{\mathcal{F}}(q(\lambda)) \geq 2$ and $\operatorname{rank} W_{\mathcal{F}^{\prime}}\left(q^{\prime}(\lambda)\right)=2$. Since $a d-b c \neq 0$, clearly $\operatorname{rank} W_{\mathcal{F}}(q(\lambda)) \geq 2$ and since $\lambda \gg 0$, the two columns of $W_{\mathcal{F}^{\prime}}\left(q^{\prime}(\lambda)\right)$ are independent, thus $\operatorname{rank} W_{\mathcal{F}^{\prime}}\left(q^{\prime}(\lambda)\right)=2$. We complete the proof of the claim by showing that every column of $W_{\mathcal{F}}(q(\lambda))$ can be written as a linear combination of columns of $W_{\mathcal{F}^{\prime}}\left(q^{\prime}(\lambda)\right)$. Indeed,

$$
\begin{aligned}
{\left[\begin{array}{c}
-\left(a \lambda_{1}+b \lambda_{2}\right) \\
a \\
b
\end{array}\right] } & =\left(a+b \frac{\lambda_{2}}{\lambda_{1}}\right)\left[\begin{array}{c}
-\lambda_{1} \\
1 \\
0
\end{array}\right]+b\left[\begin{array}{c}
0 \\
-\frac{\lambda_{2}}{\lambda_{1}} \\
1
\end{array}\right], \\
{\left[\begin{array}{c}
-\left(c \lambda_{1}+d \lambda_{2}\right) \\
c
\end{array}\right] } & =\left(c+d \frac{\lambda_{2}}{\lambda_{1}}\right)\left[\begin{array}{c}
-\lambda_{1} \\
1 \\
0
\end{array}\right]+d\left[\begin{array}{c}
0 \\
-\frac{\lambda_{2}}{\lambda_{1}} \\
1
\end{array}\right], \text { and } \\
{\left[\begin{array}{c}
0 \\
-\frac{\lambda_{2}}{\lambda_{1}} \\
1
\end{array}\right] } & =\left[\begin{array}{c}
0 \\
-\frac{\lambda_{2}}{\lambda_{1}} \\
1
\end{array}\right] .
\end{aligned}
$$

\section{References}

Angeloni, L., and B. Cornet (2006), "Existence of financial equilibria in a multi-period stochastic economy," Advances in Mathematical Economics 8, 1-31.

Aouani, Z., and B. Cornet (2011), "Reduced equivalent form of financial structure," Journal of Mathematical Economics 47, 318-27. Aouani, Z., and B. Cornet (2009), "Existence of financial equilibria with restricted participation," Journal of Mathematical Economics 45, 772-86.

Cornet, B., and A. Ranjan (2012a), "Existence of financial quasi-equilibria with restricted participation," Working Paper, Université Paris 1.

Cornet, B., and A. Ranjan (2012b), "Existence of financial equilibria in a multi-period economy with restricted participation," Working Paper, Université Paris 1.

Debreu, G. (1959), Theory of Value, New York: Wiley.

Florenzano, M., and P. Gourdel (1994), “T-period economies with incomplete markets,” Economic Letters 44, 91-7.

Magill, M., and M. Quinzii (1996), Theory of Incomplete Markets, Cambridge, MA: MIT Press. 